\title{
Budget Impact of a Steroid-Eluting Sinus Implant Versus Sinus Surgery for Adult Chronic Sinusitis Patients with Nasal Polyps
}

\author{
Frank R. Ernst, PharmD, MS; Ryan J. Imhoff, MS; Adam DeConde, MD; and R. Peter Manes, MD
}

\begin{abstract}
BACKGROUND: A corticosteroid-eluting sinus implant was recently approved by the FDA as a drug to treat adult patients with nasal polyps who have undergone previous endoscopic sinus surgery (ESS) of the ethmoid sinuses. ESS is performed in an operating room under general anesthesia, whereby diseased tissue and bone are removed to provide improved drainage. ESS typically involves dissection of 1 or more of the 4 paired sinus cavities (maxillary, ethmoid, sphenoid, or frontal). The implant, containing $1,350 \mathrm{mcg}$ of mometasone furoate, is inserted by a physician in an office setting and offers controlled localized release of corticosteroid to the polypoid sinus tissue. The implant has demonstrated significant improvements in clinical testing; however, little research has been conducted on its economic impact.
\end{abstract}

OBJECTIVE: To evaluate and quantify the budget impact to a commercial payer of using this implant instead of ESS in patients with nasal polyps after a previous ESS. Since essentially all patients with recurrent nasal polyps after ESS are patients with chronic sinusitis (CS) diagnosis, this study also identified patients with CS with nasal polyposis (CSwNP) for consistency with the patient population studied in clinical trials evaluating the implant.

METHODS: A budget impact analysis was conducted from a U.S. commercial payer perspective over a 1-year time horizon with patients who received the implant or revision ESS. Primary outcomes of interest were annual total and per-member per-month (PMPM) direct health care costs. Costs were estimated using a decision analysis model, assuming $50 \%$ implant utilization as an alternative to revision ESS in eligible patients, with other levels $(25 \%$, $75 \%$ ) also considered. The model utilized the results of a recently published analysis of 86,052 patients in the Blue Health Intelligence database, results from published clinical trials evaluating the implant, a literature review, and published Medicare national payment amounts.

RESULTS: A commercial health plan with 1 million members could anticipate $1,000 \mathrm{CSwNP}$ patients as candidates for receiving the implant or revision ESS. Estimated direct treatment costs for refractory CSwNP using only revision ESS are \$11.03 million (\$0.92 PMPM). If the implant replaced surgery in $50 \%$ of cases and if $63 \%$ those patients received a second treatment with the implant during the year, the estimated total cost savings would be $\$ 2.56$ million (\$0.21 PMPM). Cost savings associated with using the implant changed to \$0.11 PMPM and \$0.32 PMPM with implant adoption of $25 \%$ and $75 \%$, respectively.

CONCLUSIONS: In a large commercially insured U.S. population, annual revision ESS costs are substantial. Using the implant instead of revision ESS could result in considerable cost savings for payers at various levels of adoption.

J Manag Care Spec Pharm. 2019;25(8):941-50

Copyright $\odot 2019$, Academy of Managed Care Pharmacy. All rights reserved.

\section{What is already known about this subject}

- Among patients with chronic sinusitis with nasal polyposis (CSwNP), nasal polyposis may recur within a year after endoscopic sinus surgery (ESS) in up to $38 \%$ of cases, leading to higher surgical costs among CSwNP patients compared with chronic sinusitis patients without nasal polyposis.

- In December 2017, the FDA approved a corticosteroid-eluting (mometasone furoate) sinus implant indicated for the treatment of nasal polyps in patients aged $\geq 18$ years who had ESS.

- The implant has been found to improve patient-reported symptom measures, decrease ethmoid sinus obstruction and bilateral polyp grade, and ultimately reduce the proportion of patients indicated for revision surgery.

\section{What this study adds}

- For a U.S. health plan of 1 million members, the robust model estimated that 1,000 patients would meet the criteria to receive either a revision ESS or implant.

- Comparing the current environment, where the implant is unavailable, to a new environment, where $50 \%$ of eligible patients would receive it, direct expected annual health care cost savings for the plan were estimated to be more than $\$ 2.5$ million, corresponding to a decrease of $\$ 0.21$ per member per month (PMPM). - In hypothetical low (25\%) and high (75\%) uptake scenarios, the estimated savings were more than $\$ 1.2$ million (\$0.11 PMPM) and $\$ 3.8$ million (\$0.32 PMPM), respectively.

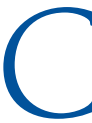

hronic sinusitis (CS) is a common disease in the United States. Prevalence estimates vary widely, from $2 \%-16 \%$, because of differences in CS diagnostic criteria. ${ }^{1-5}$ Of these patients, an estimated 19\%-55\% have CS with nasal polyposis (CSwNP), a condition associated with higher costs and utilization of office visits, medications, and procedures compared with CS patients without nasal polyposis. ${ }^{6-12}$ This is partly attributable to increased symptom severity and lower quality of life among CSwNP patients and an increased likelihood of recurrence and progression of the intrinsic inflammatory process of CSwNP..$^{13-15}$

Current guidelines recommend a variety of medications as first-line therapies for CSwNP patients, including intranasal, topical, and oral (systemic) corticosteroids, antihistamines, and nasal saline irrigations. ${ }^{16,17}$ In cases where symptoms are 


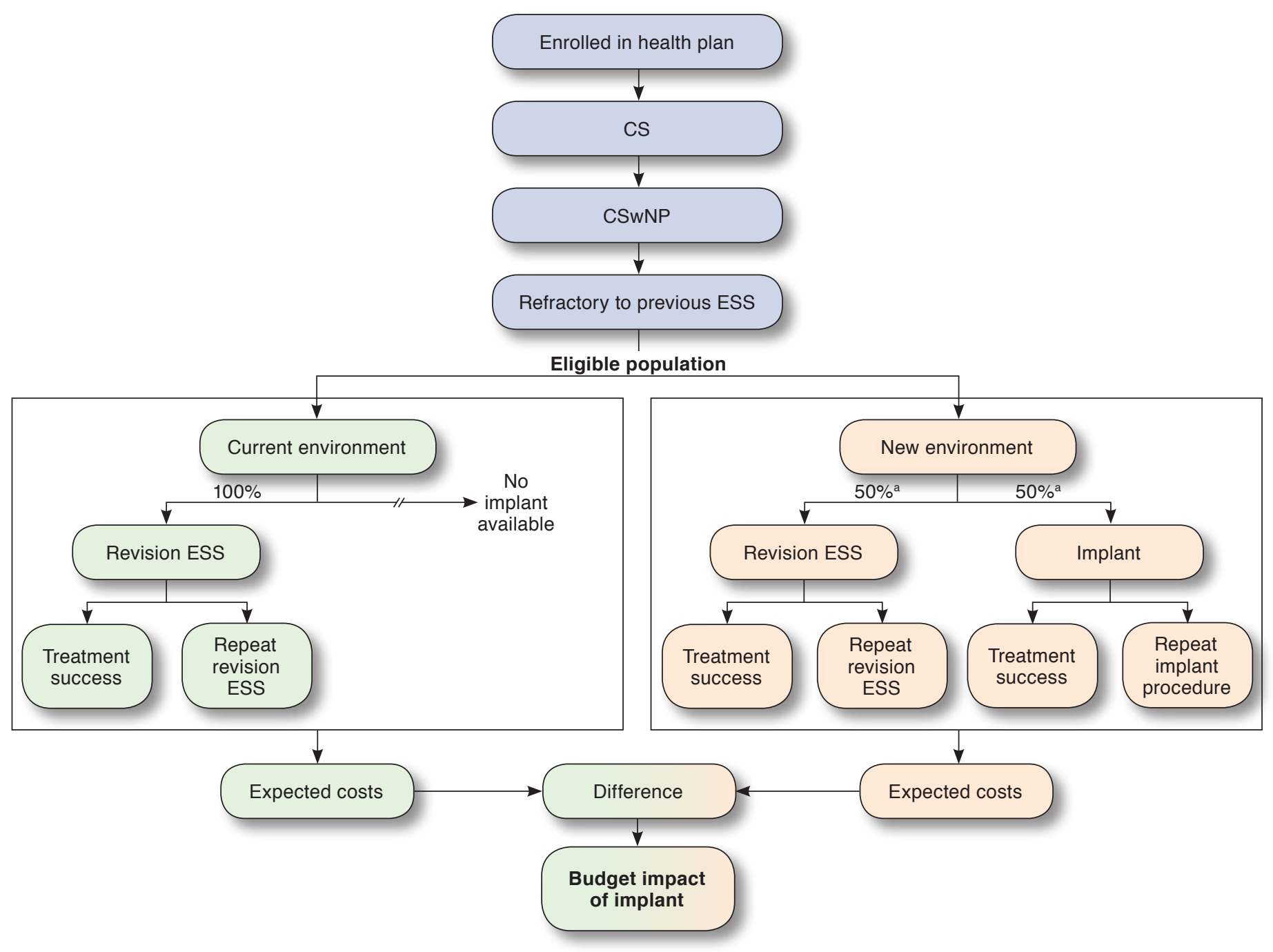

aThe base case model assumed that in the new scenario, implant uptake was 50\%. In the scenario analysis, 25\% and 75\% uptake rates were also modeled. $C S=$ chronic sinusitis; $C S w N P=$ chronic sinusitis with nasal polyposis; ESS=endoscopic sinus surgery.

refractory to medical therapy, endoscopic sinus surgery (ESS) is recommended. ${ }^{16}$

Adding to the potential concerns over the economics of health care resource utilization for patients with CS, patients may experience recurrence of CS symptoms that can require a revision ESS procedure. Indications for revision ESS include incomplete previous surgery, complications of previous surgery, recurrent or persistent sinus disease, and histological evidence of neoplasia. These criteria are not absolute, and the decision to reoperate is most often based on clinician judgment, clinician experience, and patient preference. ${ }^{18}$ Among patients with CSwNP, the nasal polyposis recurrence rate at
6 months after ESS is 35\%, increasing to 38\% at 12 months. ${ }^{19}$ This disproportionately higher risk is reflected in increased surgical costs among CSwNP patients compared with CS patients without nasal polyposis. ${ }^{20}$

Recently, the U.S. Food and Drug Administration (FDA) approved a new drug, the SINUVA Sinus Implant (Intersect ENT, Menlo Park, CA), a corticosteroid-eluting (1,350 mcg of mometasone furoate) implant indicated for the treatment of nasal polyps in patients aged $\geq 18$ years who had ESS. ${ }^{21}$ The implant was designed for insertion in an office setting by physicians trained in otolaryngology and can be removed by day 90 or earlier, at the physician's discretion 
using standard surgical instruments. Constructed of bioabsorbable polymers, the implant works by expanding within the ethmoid sinus cavity to deliver the corticosteroid directly into the polyposis for up to 90 days.

The implant is the first of this new drug category offering localized, controlled drug delivery directly to the polypoid sinus tissue and has been studied in 4 clinical trials with followup ranging from 3-6 months. ${ }^{22-26}$ The pivotal trial, RESOLVE II, was a 300-patient randomized, blinded, controlled clinical study, and the 3 other trials (a feasibility study; a pharmacokinetic study; and a 100-patient randomized, blinded, controlled study called RESOLVE) provided supportive safety and efficacy data. All patients enrolled in the randomized trials were considered by their clinicians to be candidates for revision sinus surgery. In RESOLVE II, the implant was found to improve patient-reported symptom measures, decrease ethmoid sinus obstruction and bilateral polyp grade, and ultimately reduce the proportion of patients indicated for revision surgery. ${ }^{26}$

The objective of this economic analysis was to estimate the budgetary effects of using the implant instead of a revision ESS procedure in CSwNP patients who have undergone a previous ESS and are candidates for revision surgery due to recurrent polyposis, despite attempts with medical management. Given the availability of the implant in the United States, the model adopted the perspective of a commercial third-party U.S. payer.

\section{Methods}

A budget impact analysis was performed to estimate the potential cost savings of adopting the implant. The expected budget impact was estimated over a l-year time horizon. To estimate the expected costs for each treatment strategy, a decision tree analytic model was developed. The decision tree method was chosen due to the short time horizon, the cohort-level analysis, and the straightforward nature of the outcomes. ${ }^{27}$ The model was developed based on previously collected or published data, and as such, it was exempt from institutional review board review.

The analysis follows the International Society for Pharmacoeconomics and Outcomes Research Principles of Good Practice Guidelines. ${ }^{27,28}$ All modeling was performed in Microsoft Excel (Microsoft Corporation, Redmond, WA) and validated in TreeAge Pro 2017 (TreeAge Software, Williamstown, MA).

\section{Model Structure}

This model simulates the clinical management of patients aged $\geq 18$ years with nasal polyps who experience a recurrence of symptoms following an ESS procedure. All patients are assumed to receive either the implant or revision ESS procedure (Figure 1). This analysis evaluates 2 scenarios: (1) the current environment where the implant is not available and thus all patients receive a revision ESS procedure, and (2) a new environment where the implant is available as an alternative to revision ESS. Since the implant uptake is not yet known, the base-case model assumed a 50\% substitution of revision ESS with the implant (new environment). This assumption was explored in a scenario analysis examining results with 25\% and $75 \%$ uptake. The primary outcome of the model is the estimated budget impact in terms of change in total and permember per-month (PMPM) treatment costs across the entire health plan.

Multiple data sources were used to inform input model values. The primary source for ESS costs and revision ESS rates was a recently published analysis that utilized the Blue Health Intelligence (BHI) commercial payer database to evaluate 86,052 patients with an ESS procedure (based on ethmoidectomy Current Procedural Terminology [CPT] codes) for CS in 2012-2015. ${ }^{20}$ In addition to the annual costs described by Hunter et al. (2018), additional unpublished costs were derived from the BHI database for the index ESS visit and the 1- to 90-day post-index period. Since the implant was approved by the FDA in December 2017 and the BHI database did not therefore include product-specific information on the implant, all model parameters that were not available from the BHI analysis were obtained from a best evidence literature review of English language articles and government-sponsored reports indexed in PubMed, along with published Medicare national payment amounts (NPAs) for relevant CPT codes.

In assessing the published evidence for the implant, 2 data points were of relevance to the model: the rate of recurrent sinusitis and the proportion of patients still indicated for revision ESS at study completion. Conservatively, we used results from the RESOLVE randomized trial that evaluated 100 patients who received either implants or a sham procedure. The reasons were 2-fold: the rate of recurrent sinusitis was highest in this study, and patients were followed through 6 months, meaning that the proportion of patients still indicated for revision ESS was higher compared with other studies. ${ }^{24,25}$

\section{Population}

A health plan of 1 million hypothetical adult members was modeled. Since essentially all patients with recurrent nasal polyps after ESS have a CS diagnosis, our study identified patients with CSwNP for consistency with the patient population studied in clinical trials evaluating the implant. Thus, determining the proportion of members who would likely have nasal polyps required combining CS prevalence information with nasal polyposis as a subset of CS. CS prevalence was informed using data extracted from the literature, with a pooled estimate (2.94\%) derived from a sample-weighted average of several studies (Table 1). ${ }^{1,2,4,5}$ The proportion of CS patients with nasal polyps (41\%) was populated from the BHI database study results. ${ }^{20}$ In order to estimate the proportion of patients eligible to receive the implant or a revision ESS, which was defined as CSwNP patients who were refractory to medical treatment and 


\section{TABLE 1 Parameter Values}

\begin{tabular}{|c|c|c|c|c|}
\hline Parameter & Base Case & Low $(-25 \%)$ & High $(+25 \%)$ & Reference \\
\hline \multicolumn{5}{|l|}{ Population inputs } \\
\hline CS prevalence, $\%$ & 2.9 & 2.2 & 3.7 & Bhattacharyya 2011, ${ }^{1}$ Bhattacharyya $2012,{ }^{2}$ Pleis $2009,{ }^{4}$ Shashy $2004^{5}$ \\
\hline Proportion of CS with NP, \% & 41.0 & 30.8 & 51.3 & Hunter $2018^{20}$ \\
\hline CSwNP ESS revision rate, \% & 8.3 & 6.2 & 10.4 & Hunter $2018^{20}$ \\
\hline \multicolumn{5}{|l|}{ Drug implant inputs } \\
\hline Implant product cost, $\$$ & 2,550 & 1,913 & 3,188 & Price reported to Red Book by manufacturer \\
\hline Implant procedure cost \$ & 394 & 296 & 493 & CMS 201729 (CPT 31237) \\
\hline 3-month follow-up cost, $\$$ & 644 & 483 & 805 & 2 office visits with endoscopy at each \\
\hline Office visit (each), \$ & 110 & - & - & CMS 201729 (average of CPT 99213-99215) \\
\hline Endoscopy (each), \$ & 212 & - & - & CMS 201729 (CPT 31231) \\
\hline 3-month acute sinusitis rate, $\%$ & 32.0 & 24.0 & 40.0 & Han 201424 \\
\hline Sinusitis treatment cost, $\$$ & 134 & 101 & 168 & Office visit and oral antibiotic \\
\hline Office visit, \$ & 110 & - & - & CMS $2017^{29}$ (average of CPT 99213-99215) \\
\hline Amoxicillin 875 mg, 14-day course, $\$$ & 24 & - & - & Pleis $2009^{4}$ \\
\hline 6-month retreatment rate ${ }^{a}, \%$ & 62.9 & 47.2 & 78.6 & Lavigne 2014,22 Forwith $2016^{25}$ \\
\hline \multicolumn{5}{|l|}{ Revision ESS inputs } \\
\hline Presurgical cost, $\$$ & 419 & 314 & 524 & Office visit with labs, nasal CT, and ECG \\
\hline Office visit, \$ & 110 & - & - & CMS $2017^{29}$ (average of CPT 99213-99215) \\
\hline CBC lab & 26 & - & - & Labcorp $2013^{32}$ \\
\hline BMP lab & 31 & - & - & Labcorp $2013^{32}$ \\
\hline Nasal CT scan & 236 & - & - & CMS 201729 (CPT 70480) \\
\hline ECG & 17 & - & - & CMS 201729 (CPT 93000) \\
\hline Procedure cost, $\$$ & 9,398 & 7,048 & 11,747 & Hunter $2018^{20}$ \\
\hline 3-month follow-up cost, \$ & 1,038 & 779 & 1,298 & Hunter $2018^{20}$ \\
\hline 6-month retreatment rate, $\%$ & 1.6 & 1.2 & 2.0 & Hunter $2018^{20}$ \\
\hline \multicolumn{5}{|c|}{$\begin{array}{l}\text { Note: Where multiple values were found in the literature for a given parameter, a sample-weighted average was calculated. } \\
\text { according to the December } 2017 \text { prescribing information for the implant, retreatment had not yet been studied. An assumed retreatment rate was included solely for the } \\
\text { purposes of economic modeling. } \\
B M P=\text { blood metabolic panel; } C B C=\text { complete blood count; } C P T=C \text { Current Procedural Terminology; } C S=\text { chronic sinusitis; } C S w N P=\text { chronic sinusitis with nasal polyposis; } \\
C T=\text { computed tomography; ECG =electrocardiogram; ESS=endoscopic sinus surgery; NP=nasal polyps. }\end{array}$} \\
\hline
\end{tabular}

previous ESS and who elected to undergo a revision ESS, the revision rate among CSwNP patients was calculated from the BHI analysis (8.3\%).

\section{Clinical Data}

For the revision ESS group, health care utilization, including routine preprocedural and follow-up care, along with any complications or adverse events (AEs) that required medical intervention (e.g., office visit, medication, and procedure; Table 1) during the 3-month postprocedural period, was evaluated. In addition, revision ESS rates within 6 months of the initial procedure were utilized.

For the implant group, it was assumed, based on clinical expertise, that no preprocedural work-up would be required due to the minimally invasive nature of the procedure and the safety of the drug implant. It was also assumed, based on expertise of the practicing clinician researchers, that patients would be seen by their physicians for 2 follow-up office visits and would receive a nasal endoscopy procedure during each encounter. Consistent with the RESOLVE and RESOLVE II clinical trials and the bilateral nature of sinonasal polyposis, it was assumed that all patients receive 2 implants per encounter (1 per ethmoid sinus cavity; Table 1). In multiple clinical trials, complications and AEs directly attributed to the implant were infrequent and typically did not require medical intervention (e.g., nasal discomfort and nasal odor). Therefore, these AEs were not included in the model, since cost of care could not reasonably be assigned to them. The most frequently reported AE overall, and not necessarily directly attributed to the implant, was recurrent sinusitis (acute or chronic). Conservatively, the highest reported rate, which was observed in the RESOLVE study, was used as the model input for this parameter. In that study, sinusitis occurred in $32 \%$ of treated patients compared with $45 \%$ of controls within 90 days. ${ }^{24}$ Other AEs were relatively infrequent and balanced between treatment and control groups. Therefore, acute sinusitis was considered in this model, and it was assumed to require a physician office visit and an appropriate course of antibiotics. A sample-weighted pooled average of 2 clinical trials (the RESOLVE trial and a study by Lavigne et al., 2014) reporting the proportion of patients still 
eligible for revision 6 months after receiving an implant was used to estimate the 6-month retreatment rate $(62.9 \%){ }^{22,25}$ This is likely an overestimation, since this assumes that all patients indicated for a second procedure elect to receive one.

For the revision ESS group, it was assumed that the preprocedural work-up would include a physician office visit with orders for the following laboratory tests: complete blood count, basic metabolic panel, nasal computed tomography (CT) scan, and electrocardiogram (ECG; Table 1). Aggregate diseaserelated 3-month postprocedural costs were available from the analysis of BHI claims data for this group, so it was not necessary to consider individual AEs and components of follow-up care (such as acute sinusitis and nasal debridement), as was done for the implant group. ${ }^{20}$ The 6 -month revision ESS rate estimate from the BHI analysis was used.

\section{Cost Data}

The costs for both groups fell into 3 categories: preprocedural costs, procedural costs, and follow-up costs. Table 1 shows all of the parameter values. For the implant, it was assumed, based on an understanding of clinical practices, that no preprocedural work-up was required; thus, no costs in this category would be incurred. Estimated procedural costs included the cost of the product as reported to RED BOOK by the manufacturer and the cost of the implantation procedure (CPT code 31237), which was obtained from the NPA. ${ }^{29}$ During follow-up, office visit costs (averaged from CPT codes 99213-99215) and nasal endoscopy costs (CPT code 31231) were also obtained from the NPA. Since all procedures in this model were assumed to be bilateral, $150 \%$ of the reported NPA was used where appropriate, per current billing standards. ${ }^{30}$ The antibiotic cost for treating acute sinusitis was estimated as the average wholesale price of a 14-day course of amoxicillin ( $875 \mathrm{mg}$ ) as reported by LexiComp Online. ${ }^{31}$

For revision ESS, preprocedural blood work costs were estimated from the LabCorp Fee Schedule, while CT scan (CPT code 70480) and ECG (CPT code 93000) costs were obtained from NPAs. ${ }^{32}$ The revision ESS procedure visit cost was estimated to be the average disease-related medical and prescription costs for an ESS visit in the BHI database analysis. ${ }^{20}$ Follow-up costs after revision were also estimated from the BHI analysis and were defined as the average total disease-related medical and prescription drug costs incurred within 90 days after the ESS procedure.

While repeat administration of the implant had not been studied as of the time of its approval by the FDA in 2017, an assumed retreatment rate was included in our analysis for the purposes of economic modeling. For patients receiving either an implant or a revision ESS, it was assumed that any further retreatments would be of the same type. In other words, if a patient received a revision ESS procedure and experienced another recurrence, they would undergo a second revision
ESS procedure rather than switch to an implant and vice versa. Additionally, further re-treatment costs were assumed to include the same preprocedural, procedural, and follow-up costs associated with the first revision procedure.

\section{Sensitivity Analysis}

One-Way Sensitivity Analysis. A one-way deterministic sensitivity analysis was conducted by varying each model parameter individually and observing the effect on the results in order to assess the impact of the individual model parameters and the robustness of the findings. ${ }^{33}$ Since the standard error values for population means from the BHI analysis were relatively small, all parameters were changed by $\pm 25 \%$ in the sensitivity analysis to allow for a more rigorous and conservative test of the results. ${ }^{20}$

Probabilistic Sensitivity Analysis. A 100,000-trial Monte Carlo probabilistic sensitivity analysis was performed to further evaluate the robustness of the results by testing second-order uncertainty. ${ }^{33}$ This sensitivity analysis was used to estimate the proportion of simulated trials for which the implant resulted in cost savings. For all variables, triangular distributions were assumed, with the default values used as the most likely values and default values $\pm 25 \%$ used as the minimum and maximum values. As described for the one-way sensitivity analysis, this was chosen as a rigorous test of the uncertainty in the model. Triangular distributions were deemed appropriate because we were confident in the point estimates representing the most likely value and $\pm 25 \%$ representing conservative minimum and maximum values.

\section{Scenario Analysis}

In the base-case model, an uptake of 50\% utilization of the implant instead of revision ESS was estimated and compared against the current environment without the implant as a treatment option. Since the actual uptake of the implant is unknown, a scenario analysis was conducted to determine the effect of changing this assumption. Two scenarios were considered: a low implant uptake of $25 \%$ and a high implant uptake of $75 \%$. Changes in total and PMPM health care cost savings were determined under each scenario.

\section{Results}

\section{Base Case}

In the base-case scenario, the average expected annual cost of treating a patient with revision ESS was $\$ 11,029$. By comparison, the expected annual cost of treating a patient with the implant was $\$ 5,916$, representing savings of $\$ 5,113$ per patient treated with the implant instead of revision ESS. For a U.S. commercial third-party payer with a health plan of 1 million members, the model estimated that 1,000 patients would meet the criteria to receive either a revision ESS or the implant. In 
FIGURE 2 Impact of Corticosteroid Implant on Plan-Level Health Care Costs for CSwNP Patients

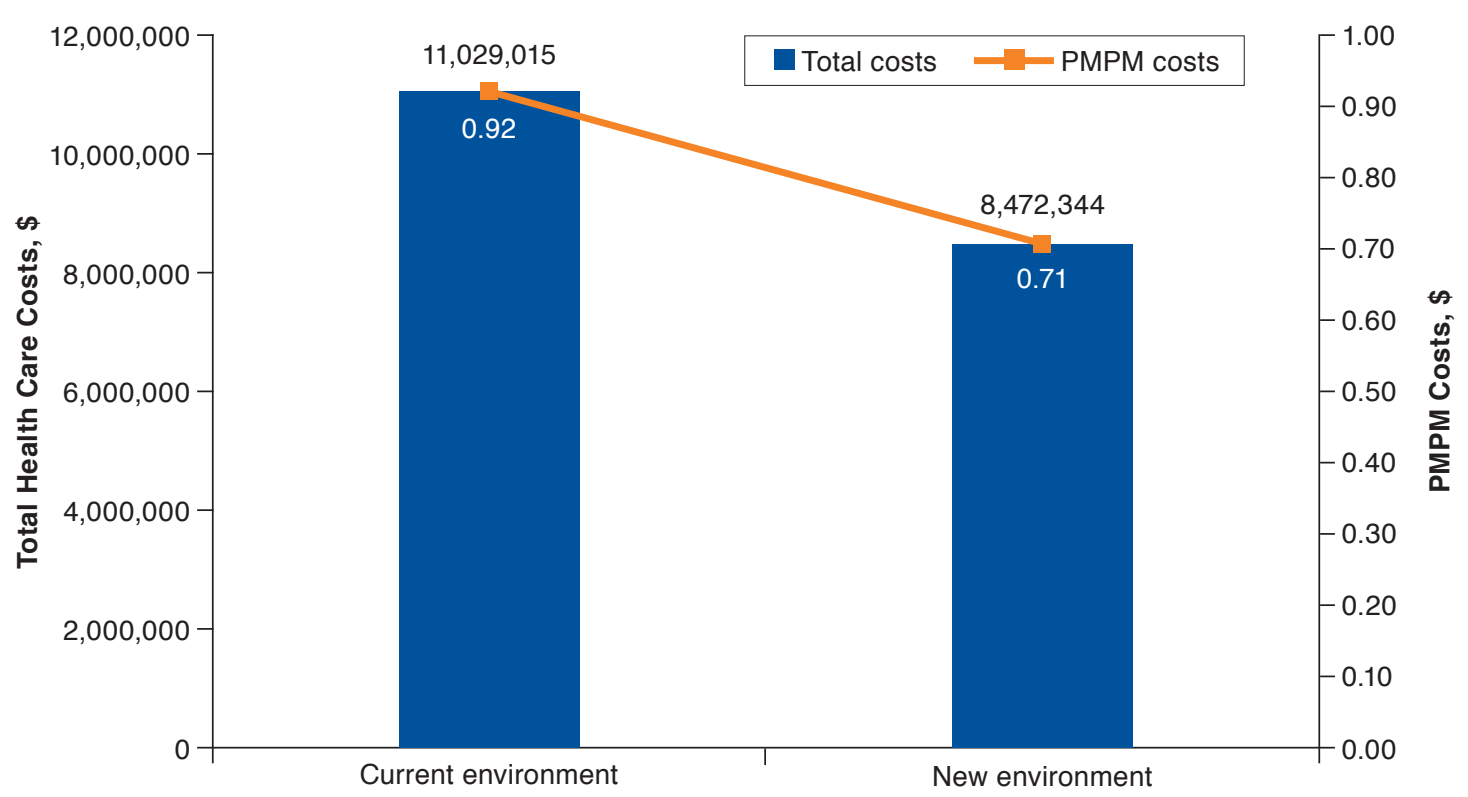

Notes: Total health care costs of treating CSwNP for a commercial third-party payer with 1,000,000 members over a 1-year time horizon. The model assumed 1,000 patients were treated and in the current environment, 100\% of patients received a revision ESS procedure, whereas in the new environment, 50\% of patients received bilateral implants instead. Values above each bar correspond to total health care costs (left axis). Values below the top of each bar correspond to the PMPM cost (right axis). $C S w N P=$ chronic sinusitis with nasal polyposis; $P M P M=$ per-member per-month .

the current environment where the implant is unavailable, total expected annual health care costs were estimated at $\$ 11,029,015$ (\$0.92 PMPM; Figure 2). In a new environment where the implant would be used for $50 \%$ of patients, expected annual health care costs were estimated to be $\$ 8,472,344$ (\$0.71 PMPM). Thus, the expected budget impact of the implant was estimated to be savings of $\$ 2,556,671$ ( $\$ 0.21$ PMPM) each year. Despite an assumed 63\% rate of retreatment at 6 months, lower estimated procedural costs appear to drive substantial cost savings for the payer.

\section{Sensitivity Analysis}

One-Way Sensitivity Analysis. The one-way sensitivity analysis showed the results of the model to be robust to changes in parameter values within $\pm 25 \%$ of the base-case value, with cost savings related to the use of the implant retained in all instances (Figure 3). The model was most sensitive to the revision ESS procedure costs, the number of eligible patients in the plan (a change in any of the default population prevalence rate variables), and the implant product cost (if that cost were hypothetically allowed to vary).

Probabilistic Sensitivity Analysis. In the probabilistic sensitivity analysis, the use of the implant was estimated to result in cost savings in $100 \%$ of the Monte Carlo simulation trials.
Moreover, in $46.6 \%$ of trials, estimated cost savings associated with the use of the implant were greater than the base-case result, ranging from $\$ 480,773$ to $\$ 6,654,284$. These results indicate that the model was highly robust to variations in basecase parameter values.

\section{Scenario Analysis}

In the low uptake scenario, where $25 \%$ of patients would receive an implant instead of revision ESS, the estimated budget impact of implant use was savings of $\$ 1,278,336$ ( $\$ 0.11$ PMPM; Figure 4). Conversely, in the high uptake scenario, where $75 \%$ of patients receive an implant instead of revision ESS, estimated savings were $\$ 3,835,007$ ( $\$ 0.32$ PMPM).

\section{Discussion}

In an atmosphere where the United States is facing increasing health care costs, it is becoming more important to demonstrate both the clinical benefit and economic value of new and innovative medical technologies. Findings from clinical trials assessing the efficacy of a new drug implant for the sinuses have been very encouraging, with reductions in ethmoid sinus obstruction, nasal polyp grade, patient-reported symptom scores, and candidacy for a subsequent revision ESS. $22,24,25$ 


\section{R-ESS procedure cost}

Eligible number of patients in plan

Implant product cost

Implant retreatment rate

R-ESS follow-up cost

Implant follow-up cost

Implant placement cost

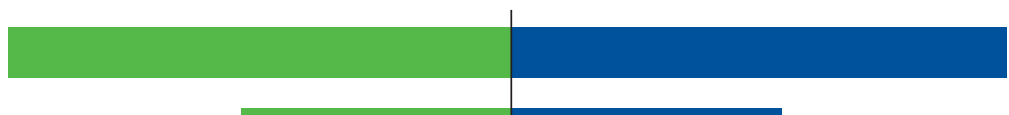

R-ESS presurgery cost

R-ESS retreatment cost

Acute sinusitis rate/cost

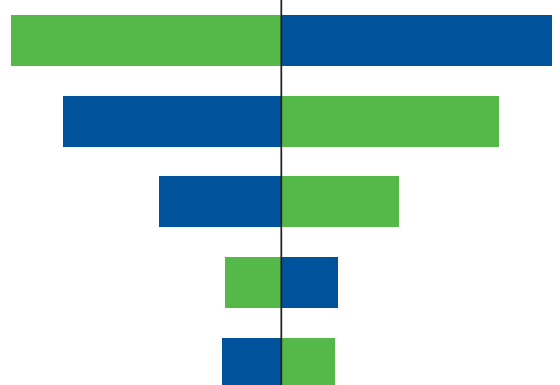

(2)

1,000

2,000

3,000

4,000

Total Cost Savings of New vs. Current Environment

(thousands of dollars)

Note: The center axis of the tornado diagram corresponds to the base-case result of $\$ 2,556,671$ in total estimated savings for a commercial, third-party payer. "Eligible number of patients in plan" combines the following variables: health plan size, CS prevalence, proportion of patients with CSwNP, and proportion of patients with CSwNP who have undergone a revision ESS procedure. These variables were combined to simplify the analysis because changing any of them would result in an equal change in the number of eligible patients.

$C S=$ chronic sinusitis; $C S w N P=$ chronic sinusitis with nasal polyposis; $R$-ESS = revision endoscopic sinus surgery.

This model found that in a hypothetical, commercial thirdparty health plan with 1 million members, 1,000 patients could have recurrent CSwNP following ESS. Our study findings suggest that if the current environment, where no patients are receiving the implant, changed to $50 \%$ of patients receiving implants instead of revision ESS, estimated cost savings of $\$ 2,556,671$ (\$0.21 PMPM) would result. These cost savings would largely be driven by substantially lower procedurerelated costs associated with the implant. The total estimated annual costs per patient receiving revision ESS were $\$ 11,029$, whereas implant-related total costs were nearly half that amount at $\$ 5,916$. Even if a patient would receive implants twice in 1 year, the total estimated costs of $\$ 7,263$ still resulted in large savings per patient.

Since the implant was not launched in the United States until early 2018, assumptions were based on information presented in clinical trials and on expert opinion of physicians practicing in the field of rhinology. One assumption is that the patient would not switch to an alternative treatment if the treatment (revision ESS or implant) failed. Rather, the model assumes that they would undergo a repeat of their initial procedure. If patients who experienced disease recurrence after an implant elected to undergo ESS rather than a second implant, cost savings could be less, since ESS is costlier than the implant procedure. Conversely, if patients who experience disease recurrence after a revision ESS procedure elected to receive the implant rather than a second revision ESS, cost savings could be even greater, since surgery always costs more than the implant, according to this model. The latter scenario might be more common given the less-invasive nature of the office-based procedure compared with ESS.

\section{Strengths}

A strength of this study is its reliance on estimates from a large real-world analysis for prevalence rates and summary cost data on revision ESS. ${ }^{20}$ Thus, it captures real-world utilization, AEs, and complications that particularly affect a commercial payer's budget. Clinical outcomes data for the implant were derived 


\section{FIGURE 4 Scenario Analysis: Impact of Corticosteroid Implant Uptake on Cost Savings}

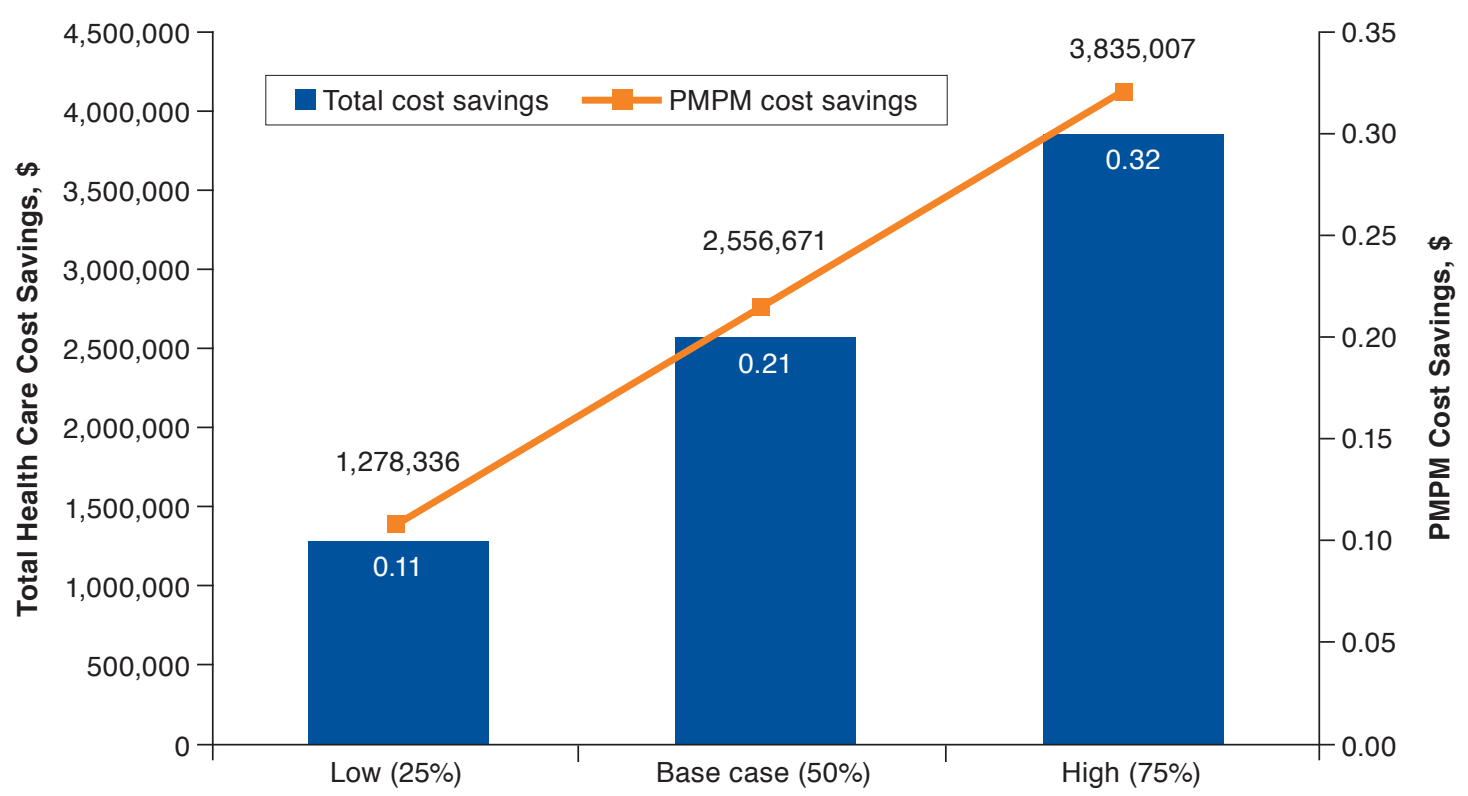

Note: Values above each bar correspond to total health care cost savings (left axis). Values below the top of each bar correspond to the PMPM cost savings (right axis). $P M P M=$ per-member per-month .

from studies published in peer-reviewed literature, providing reliable estimates. Pairing these estimates with publicly available cost data and current physician- and surgeon-level coding and billing practices helped best ensure accurate estimates of treatment costs.

To ensure that the results were robust to these assumptions and the default values used to populate the parameters, extensive sensitivity and scenario analyses were also conducted. To be conservative and more aggressively test the results of the study, low and high values were calculated from $\pm 25 \%$ of the base-case value, rather than using \pm standard error, which would have evaluated a much smaller range of values. Moreover, since implant market adoption data are not yet available for use, the treatment mix of implant versus revision ESS required estimation. Although a 50\% rate of adoption was modeled in the base-case model, the scenario analysis revealed that the estimated cost savings would decrease to $\$ 1,278,336$ (\$0.11 PMPM) if uptake were $25 \%$ or conversely increase to $\$ 3,835,007$ (\$0.32 PMPM) if uptake were $75 \%$.

\section{Limitations}

This study has some limitations that need to be considered. The model structure and data used for this analysis were designed as a simulation of real-world CSwNP care in the United States from the perspective of a commercial third-party payer. Consequently, our findings may have limited generalizability outside of these situations. However, the model structure may be adapted to other payer systems either inside or outside of the United States to assess the budget impact of the implant as an alternative to revision ESS. Additionally, this model only considered direct costs and did not account for indirect costs such as lost productivity time or effect on quality of life.

In the BHI database analysis, the cost of the surgery for patients who underwent a second surgery in the same time period was significantly higher, suggesting that the cost of revision surgery is higher than the cost of primary surgery. The implant is expected to primarily be used in patients with refractory disease as, in the RESOLVE II trial, $60 \%$ of patients had undergone 2 or more previous ESS procedures. Therefore, it is possible that the cost of revision surgery was underestimated in this study.

This analysis used the Medicare Fee Schedule to populate some of the cost parameters in the model, as opposed to average commercial reimbursement values that may be preferable. Given the conservative nature of Medicare reimbursement rates, some of the costs may be slightly underestimated. In the one-way and probabilistic sensitivity analyses, the results were shown to be robust to changes in these cost values.

Since real-world data for the implant were not available at the time of this study, to estimate costs in a real-world environment, data were adapted from randomized clinical trials and expert opinion from physicians experienced in the field 
of rhinology. Certain inputs to the model (e.g., the proportion of patients who might require a repeat implant procedure) were taken from the RESOLVE study rather than the larger pivotal RESOLVE II study, since longer-term outcomes to 6 months were available in RESOLVE.

To calculate the procedural cost of the implant, CPT billing codes were used, and these can vary depending on the patient. This study conservatively used the most expensive billing codes for the implant procedure and thus could be an overestimation of plan-level costs. Moreover, due to limited longitudinal data available for the implant in the various data sources, we did not have the ability to model longer time horizons. This lack of longitudinal data did not allow us to determine whether some patients may need the implant more frequently (e.g., every 90 days) or less frequently (e.g., once per year), or if some patients may need a series of treatments but then will not require further treatment for an extended period of time (if ever). To address these limitations and ensure this model was robust to the assumptions, rigorous sensitivity and scenario analyses were conducted.

Since the study relied significantly on the findings from a recent large retrospective BHI database analysis, the limitations of those analyses carry over as well. That study could not rule out the possibility of ESS surgery having occurred before a patient was enrolled in the insurance plans from which the BHI data were derived, nor could that study estimate revision surgery rates beyond 3 years. The current analysis used a 1-year time horizon because of the limited ability of the BHI analyses and other source studies to project reasonably beyond that time.

Additional limitations from the BHI-based study, such as lack of ability to generalize to Medicare and Medicaid patients and lack of certain clinical information, also apply by default to this study. However, the large sample size of that study was a strength carried forward into the current analyses for the applicable data elements.

\section{Conclusions}

In a large commercially insured U.S. population, current costs for patients facing revision ESS for recurrent nasal polyps could be substantial. Use of the corticosteroid implant instead of revision ESS could result in considerable cost savings for payers in a single year, due largely to much lower procedure-associated costs. The economic benefit to payers increases with higher adoption, which may have implications in considering use of the implant as step therapy before revision surgery in patients with recurrent nasal polyposis. More economic simulation research is warranted when real-world market adoption data for the implant are available.

\section{Authors}

FRANK R. ERNST, PharmD, MS, and RYAN J. IMHOFF, MS, CTI Clinical Trial and Consulting Services, Covington, Kentucky. ADAM DECONDE, MD, Department of Surgery, School of Medicine, University of California, San Diego, and R. PETER MANES, MD, Otolaryngology, Yale School of Medicine, New Haven, Connecticut.

AUTHOR CORRESPONDENCE: Frank R. Ernst, PharmD, MS, Executive Director, Real-World Evidence, CTI Clinical Trial and Consulting, 100 E. RiverCenter Blvd., Covington, KY 41011. Tel.: 513.595.9290; E-mail: fernst@ctifacts.com.

\section{DISCLOSURES}

This study was sponsored by Intersect ENT, which was involved in study design and manuscript review. Ernst and Imhoff are employed by CTI Clinical Trial and Consulting Services, which contracted with Intersect ENT to conduct this study. Ernst and Imhoff also report other financial support from Intersect ENT during the conduct of the study. DeConde reports personal fees from Intersect ENT during the conduct of the study, as well as personal fees from Optinose, Stryker Endoscopy, and Olympus, outside the submitted work. Manes reports grants from Intersect ENT during the conduct of the study, as well as grants from Optinose and Sanofi outside the submitted work.

\section{ACKNOWLEDGMENTS}

The authors gratefully acknowledge and appreciate the editorial and management support provided by Molly McKean, CPM (CTI), and James Stambaugh (Intersect ENT)

\section{REFERENCES}

1. Bhattacharyya N. Incremental health care utilization and expenditures for chronic rhinosinusitis in the United States. Ann Otol Rhinol Laryngol. 2011;120(7):423-27.

2. Bhattacharyya N. Functional limitations and workdays lost associated with chronic rhinosinusitis and allergic rhinitis. Am J Rhinol Allergy. 2012;26(2):120-22.

3. Blackwell DL, Collins JG, Coles R. Summary health statistics for U.S adults: National Health Interview Survey, 1997. Vital Health Stat 10. 2002(205):1-109.

4. Pleis JR, Lucas JW, Ward BW. Summary health statistics for U.S. adults: National Health Interview Survey, 2008. Vital Health Stat 10. 2009(242):1-157.

5. Shashy RG, Moore EJ, Weaver A. Prevalence of the chronic sinusitis diagnosis in Olmsted County, Minnesota. Arch Otolaryngol Head Neck Surg. 2004;130(3):320-23.

6. Benninger MS, Holy CE. The impact of endoscopic sinus surgery on health care use in patients with respiratory comorbidities. Otolaryngol Head Neck Surg. 2014;151(3):508-15.

7. Bhattacharyya N. Clinical and symptom criteria for the accurate diagnosis of chronic rhinosinusitis. Laryngoscope. 2006;116(7 Pt 2 Suppl 110):1-22.

8. Mascarenhas JG, da Fonseca VM, Chen VG, et al. Long-term outcomes of endoscopic sinus surgery for chronic rhinosinusitis with and without nasal polyps. Braz J Otorhinolaryngol. 2013;79(3):306-11.

9. Pearlman AN, Chandra RK, Chang D, et al. Relationships between severity of chronic rhinosinusitis and nasal polyposis, asthma, and atopy. Am J Rhinol Allergy. 2009;23(2):145-48. 
10. Soler ZM, Wittenberg E, Schlosser RJ, Mace JC, Smith TL. Health state utility values in patients undergoing endoscopic sinus surgery. Laryngoscope. 2011;121(12):2672-78.

11. Tran KN, Beule AG, Singal D, Wormald PJ. Frontal ostium restenosis after the endoscopic modified Lothrop procedure. Laryngoscope. 2007;117(8):1457-62.

12. Benninger MS, Holy CE. Endoscopic sinus surgery provides effective relief as observed by health care use pre- and postoperatively. Otolaryngol Head Neck Surg. 2014;150(5):893-900.

13. Batra PS, Tong L, Citardi MJ. Analysis of comorbidities and objective parameters in refractory chronic rhinosinusitis. Laryngoscope. 2013;123(Suppl 7):S1-11.

14. Meltzer EO, Hamilos DL, Hadley JA, et al. Rhinosinusitis: establishing definitions for clinical research and patient care. Otolaryngol Head Neck Surg. 2004;131(6 Suppl):S1-62.

15. Wu AW, Ting JY, Platt MP, Tierney HT, Metson R. Factors affecting time to revision sinus surgery for nasal polyps: a 25 -year experience. Laryngoscope. 2014;124(1):29-33.

16. Orlandi RR, Kingdom TT, Hwang PH, et al. International consensus statement on allergy and rhinology: rhinosinusitis. Int Forum Allergy Rhinol. 2016;6(Suppl 1):S22-209.

17. Rosenfeld RM, Piccirillo JF, Chandrasekhar SS, et al. Clinical practice guideline (update): adult sinusitis executive summary. Otolaryngol Head Neck Surg. 2015;152(4):598-609.

18. Tewfik MA, Desrosiers M. Indications for revision endoscopic sinus surgery. In: Kountakis SE, Jacobs J, Gosepath J, eds. Revision Sinus Surgery. Berlin, Heidelberg: Springer-Verlag Berlin Heidelberg; 2008:13-18.

19. DeConde AS, Mace JC, Levy JM, Rudmik L, Alt JA, Smith TL. Prevalence of polyp recurrence after endoscopic sinus surgery for chronic rhinosinusitis with nasal polyposis. Laryngoscope. 2017;127(3):550-55.

20. Hunter TD, DeConde AS, Manes RP. Disease-related expenditures and revision rates in chronic rhinosinusitis patients after endoscopic sinus surgery. J Med Econ. 2018:1-14.

21. SINUVA (mometasone furoate). Intersect ENT. December 2017. Available at: https://www.accessdata.fda.gov/drugsatfda_docs/label/2017/209310lbl. pdf. Accessed January 23, 2019.
22. Lavigne F, Miller SK, Gould AR, Lanier BJ, Romett JL. Steroid-eluting sinus implant for in-office treatment of recurrent nasal polyposis: a prospective, multicenter study. Int Forum Allergy Rhinol. 2014;4(5):381-89.

23. Ow R, Groppo E, Clutter D, Gawlicka AK. Steroid-eluting sinus implant for in-office treatment of recurrent polyposis: a pharmacokinetic study. Int Forum Allergy Rhinol. 2014:4(10):816-22.

24. Han JK, Forwith KD, Smith TL, et al. RESOLVE: a randomized, controlled, blinded study of bioabsorbable steroid-eluting sinus implants for in-office treatment of recurrent sinonasal polyposis. Int Forum Allergy Rhinol. 2014:4(11):861-70.

25. Forwith KD, Han JK, Stolovitzky JP, et al. RESOLVE: bioabsorbable steroid-eluting sinus implants for in-office treatment of recurrent sinonasal polyposis after sinus surgery: 6-month outcomes from a randomized, controlled, blinded study. Int Forum Allergy Rhinol. 2016;6(6):573-81.

26. Kern RC, Stolovitzky JP, Silvers SL, et al. A phase 3 trial of mometasone furoate sinus implants for chronic sinusitis with recurrent nasal polyps. Int Forum Allergy Rhinol. 2018;8(4):471-81.

27. Caro JJ, Briggs AH, Siebert U, Kuntz KM. Modeling good research practices-overview: a report of the ISPOR-SMDM Modeling Good Research Practices Task Force-1. Value Health. 2012;15(6):796-803.

28. Sullivan SD, Mauskopf JA, Augustovski F, et al. Budget impact analysis-principles of good practice: report of the ISPOR 2012 Budget Impact Analysis Good Practice II Task Force. Value Health. 2014;17(1):5-14.

29. Centers for Medicare \& Medicaid Services. Medicare Physician Fee Schedule. 2017. Available at: https://www.cms.gov/apps/physician-fee-schedule/overview.aspx. Accessed January 22, 2019.

30. Centers for Medicare \& Medicaid Services. Payment of Bilateral Procedures in a Method II Critical Access Hospital (CAH)-JA6526. 2009. Available at: https://www.cms.gov/Medicare/Medicare-Contracting/Contractor LearningResources/downloads/ja6526.pdf. Accessed January 22, 2019.

31. Lexi-Drugs. Lexi-Comp. 2017. Available at: https://online.lexi.com/lco/ action/home. Accessed January 22, 2019.

32. LabCorp. Net fee schedule. 2013. Available at: http://www.dbhds.virginia.gov/library/administration/LabCorp\%20 pricing\%20effective\%209-1-13. xls. Accessed January 22, 2019.

33. Briggs AH, Weinstein MC, Fenwick EA, Karnon J, Sculpher MJ, Paltiel AD Model parameter estimation and uncertainty: a report of the ISPOR-SMDM Modeling Good Research Practices Task Force-6. Value Health. 2012; 15(6):835-42. 